\title{
Assessment of occlusal enamel alterations utilizing depolarization imaging based on PS-OCT
}

Jonas Golde, Florian Tetschke, Robin Vosahlo, Julia Walther, Christian Hannig, et al.

Jonas Golde, Florian Tetschke, Robin Vosahlo, Julia Walther, Christian Hannig, Edmund Koch, Lars Kirsten, "Assessment of occlusal enamel alterations utilizing depolarization imaging based on PS-OCT," Proc. SPIE 11078, Optical Coherence Imaging Techniques and Imaging in Scattering Media III, 110780P (19 July 2019); doi: 10.1117/12.2527228 


\title{
Assessment of occlusal enamel alterations utilizing depolarization imaging based on PS-OCT
}

\author{
Jonas Golde $^{\mathrm{a}}$, Florian Tetschke, ${ }^{\mathrm{a}, \mathrm{b}}$, Robin Vosahlo ${ }^{\mathrm{b}}$, Julia Walther ${ }^{\mathrm{a}, \mathrm{c}}$, Christian Hannig ${ }^{\mathrm{b}}$, \\ Edmund Koch ${ }^{\mathrm{a}}$, and Lars Kirsten ${ }^{\mathrm{a}}$ \\ aTechnische Universität Dresden, Carl Gustav Carus Faculty of Medicine, Anesthesiology and \\ Intensive Care Medicine, Clinical Sensoring and Monitoring, Dresden, Germany \\ ${ }^{\text {b } T e c h n i s c h e ~ U n i v e r s i t a ̈ t ~ D r e s d e n, ~ C a r l ~ G u s t a v ~ C a r u s ~ F a c u l t y ~ o f ~ M e d i c i n e, ~ P o l i c l i n i c ~ o f ~}$ \\ Operative and Pediatric Dentistry, Dresden, Germany \\ 'Technische Universität Dresden, Carl Gustav Carus Faculty of Medicine, Department of \\ Medical Physics and Biomedical Engineering, Dresden, Germany
}

\begin{abstract}
While dental caries represents the major chronic disease of humans, visual and tactile inspection are the methods of choice in daily dental practice. Several optical technologies have been developed in recent years for the purpose of dental examination, including near-infrared light transillumination as a promising tool for the supplementation and partial replacement of radiography. In case of occlusal alterations, the incidence of surface discolorations impedes the visual assessment, whereas tactile inspection appears to yield little diagnostic information or might be detrimental. Optical coherence tomography (OCT) facilitates depth-resolved imaging with um resolution, utilizing near-infrared light, and has already shown its potential for various dental applications. We have recently demonstrated that depolarization imaging utilizing the degree of polarization uniformity (DOPU) extends those abilities by the detection of early carious lesions, as it provides an unambiguous demineralization contrast. Here, we show that this approach also enables the assessment of occlusal enamel lesions in the presence of stains, and compare PS-OCT cross sections with polarized light microscopy (PLM) images of thin sections. For tooth samples with discoloration or demineralization, respectively, PS-OCT and PLM results are in good agreement.
\end{abstract}

Keywords: polarization-sensitive optical coherence tomography, depolarization, dentistry, occlusal caries

\section{INTRODUCTION}

The prerequisite for a successful caries management is the early and precise detection of lesions. To this day, the visual and tactile inspection remains the method of choice in daily dental practice for the purpose of dental examination, which is regularly supported by bitewing radiography for registering caries extension. Considering the aim of minimizing ionizing radiation exposure and thus limited repetition, especially near-infrared light transillumination has shown its potential in clinical practice. ${ }^{1}$ In case of occlusal alterations, the incidence of surface discolorations impedes the visual assessment, whereas tactile inspection appears to yield little diagnostic information or might be detrimental, ${ }^{2}$ posing the risk of enamel breakthrough at a present hidden carious lesion. Recently, the potential of near-infrared ${ }^{3}$ imaging in assessing stained pits and fissures has been revealed, particularly highlighting the wavelength region above $1200 \mathrm{~nm}$ due to reduced absorption of the stains. ${ }^{3}$ Optical coherence tomography (OCT) facilitates depth-resolved imaging with $\mu \mathrm{m}$ resolution in the same spectral region, and is therefore a promising method for various dental applications. For occlusal caries, both intensity-based swept source $\mathrm{OCT}^{4}$ and polarization-sensitive OCT (PS-OCT) ${ }^{5}$ have been demonstrated as suitable methods for the detection of caries due to the altered enamel scattering behavior, while the latter approach exploited the additional contrast of depolarization. As this effect competes with the enamel's native birefringence in case of cross-polarized detection, ${ }^{5}$ we have recently proposed depolarization imaging utilizing the degree of polarization uniformity (DOPU) algorithm for an unambiguous demineralization contrast. ${ }^{6}$ Here, we demonstrate that this approach improved by noise-immune processing ${ }^{7}$ also enables the assessment of occlusal enamel lesions in the presence of stains, and therefore compare the PS-OCT cross sections with polarized light microscopy (PLM) images of thin sections. ${ }^{8}$

Corresponding author: Jonas Golde, jonas.golde@tu-dresden.de

Optical Coherence Imaging Techniques and Imaging in Scattering Media III, edited by

Maciej Wojtkowski, Stephen A. Boppart, Wang-Yuhl Oh, Proc. of SPIE-OSA Vol. 11078,

110780 P · C 2019 SPIE-OSA · CCC code: 1605-7422/19/\$21 · doi: 10.1117/12.2527228

Proc. of SPIE-OSA Vol. 11078 110780P-1 


\section{METHODS AND MATERIALS}

The polarization-sensitive OCT system used for this study has been described previously. ${ }^{6}$ In brief, it is based on a $1310 \mathrm{~nm}$ swept laser and a bulk optics setup, containing both Michelson interferometer and polarizationdiverse balanced detection with a single circularly polarized illumination of the sample. In comparison to Ref. 6 utilizing DOPU, here we have utilized the degree of polarization $D O P=\sqrt{\bar{Q}^{2}+\bar{U}^{2}+\bar{V}^{2}} / \bar{I}$ with a 2D Gaussian kernel for spatial averaging, which had a full width at half maximum of $3 \times 3$ with respect to the axial and lateral resolution. Furthermore, we extracted the noise parameters of both channels from a background scan, which is regularly acquired at the beginning of each measurement. Utilizing the mean powers of the noise floor $\overline{\left|n_{c o}\right|^{2}}$ and $\overline{\left|n_{\text {cross }}\right|^{2}}$, we corrected the Stokes component $I=A_{\text {co }}^{2}+A_{\text {cross }}^{2}-\overline{\left|n_{\text {co }}\right|^{2}}-\overline{\left|n_{\text {cross }}\right|^{2}}$, as it was proposed by Makita et al. ${ }^{7}$ for improved noise immunity of the DOP measurement. Due to similar noise levels for both channels in our system it was not necessary to correct $Q .{ }^{9}$ A comparison of conventional and noise-immune DOP calculation can be seen in Fig. 1, highlighting the improvements in regions of weak signal and sound enamel. The noise correction yields a higher DOP value in this case, whereas the presumably decayed region in the fissure remains at a low DOP value, thus yielding an improved depolarization and demineralization contrast.
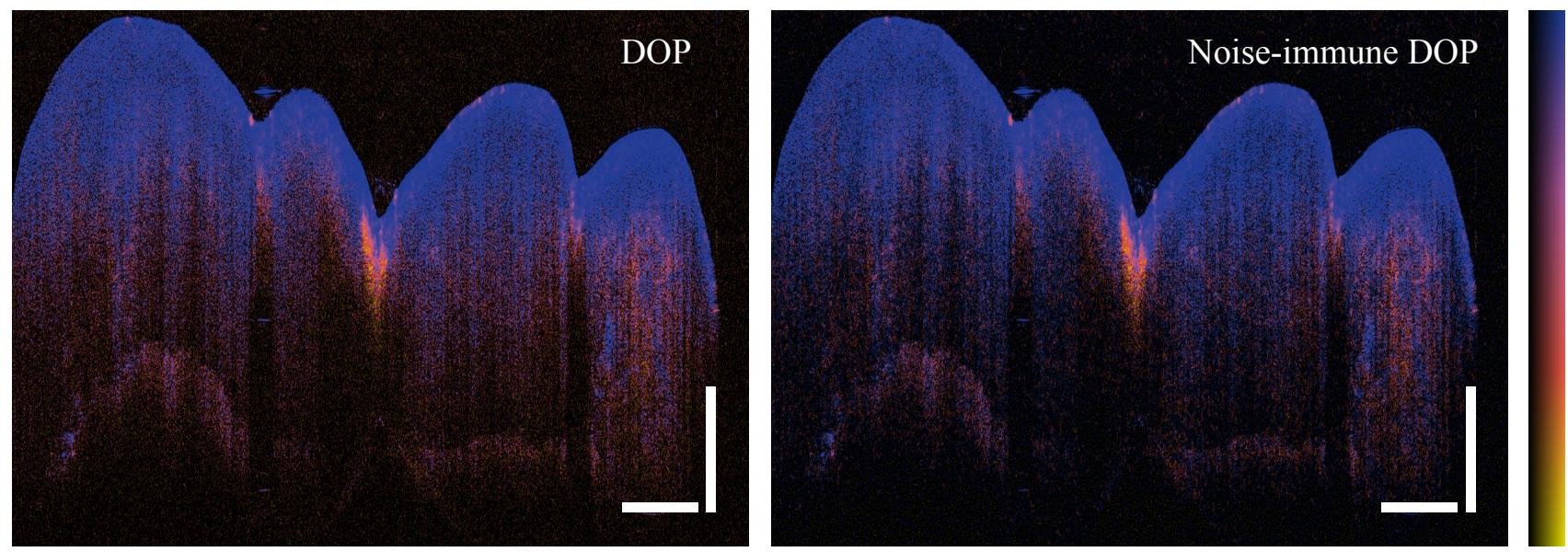

Figure 1. Comparison of conventional and noise-immune DOP processing: PS-OCT imaging of the occlusal surface shown in Fig. 3 was performed under wet conditions, the slice corresponds to an unstained region of the fissure. Depolarization visualization with intensity weighting scales from $\mathrm{DOP}=0$ (yellow) to $\mathrm{DOP}=1$ (blue). All scale bars: $1 \mathrm{~mm}$ in air.

For a more consistent representation of depolarization, we combined the calculated noise-immune DOP with high-contrast intensity images. ${ }^{10}$ Therefore, lower and upper intensity threshold were based on the extracted noise parameters, ranging from mean noise plus standard deviation to $16 \mathrm{~dB}$ above. Similarly, the intensity image range was adjusted to $60 \mathrm{~dB}$ above mean noise.

Two molar tooth samples were extracted and collected during clinical treatment. After visual inspection by an experienced dentist, PS-OCT images were acquired under wet and dry conditions. Subsequently, the teeth were cut into thin sections of about $50 \mu \mathrm{m}$ to $80 \mu \mathrm{m}$ thickness, and examined with a Leica DMRB microscope and Leica objectives with $2.5 \mathrm{x}$ and $10 \mathrm{x}$ magnification, utilizing perpendicularly adjusted polarizer and analyzer for a polarization contrast.

\section{RESULTS AND DISCUSSION}

Two molar teeth with stains and suspected lesions were selected and examined. Fig. 2a shows the occlusal surface of a molar tooth with extensive light brown discoloration and dark brown discoloration in the pits. The PLM images Fig. 2b and c confirm the suspected lesion in the fissure and the surrounding superficial demineralization of the enamel. In comparison, the intensity image (d) recorded with a wetted surface appears relatively unremarkable, whereas the depolarization representation (e) highlights the fissure due to the infiltrated caries. Contrarily, the intensity image of the dry tooth (f) may facilitate an observation of altered scattering properties 

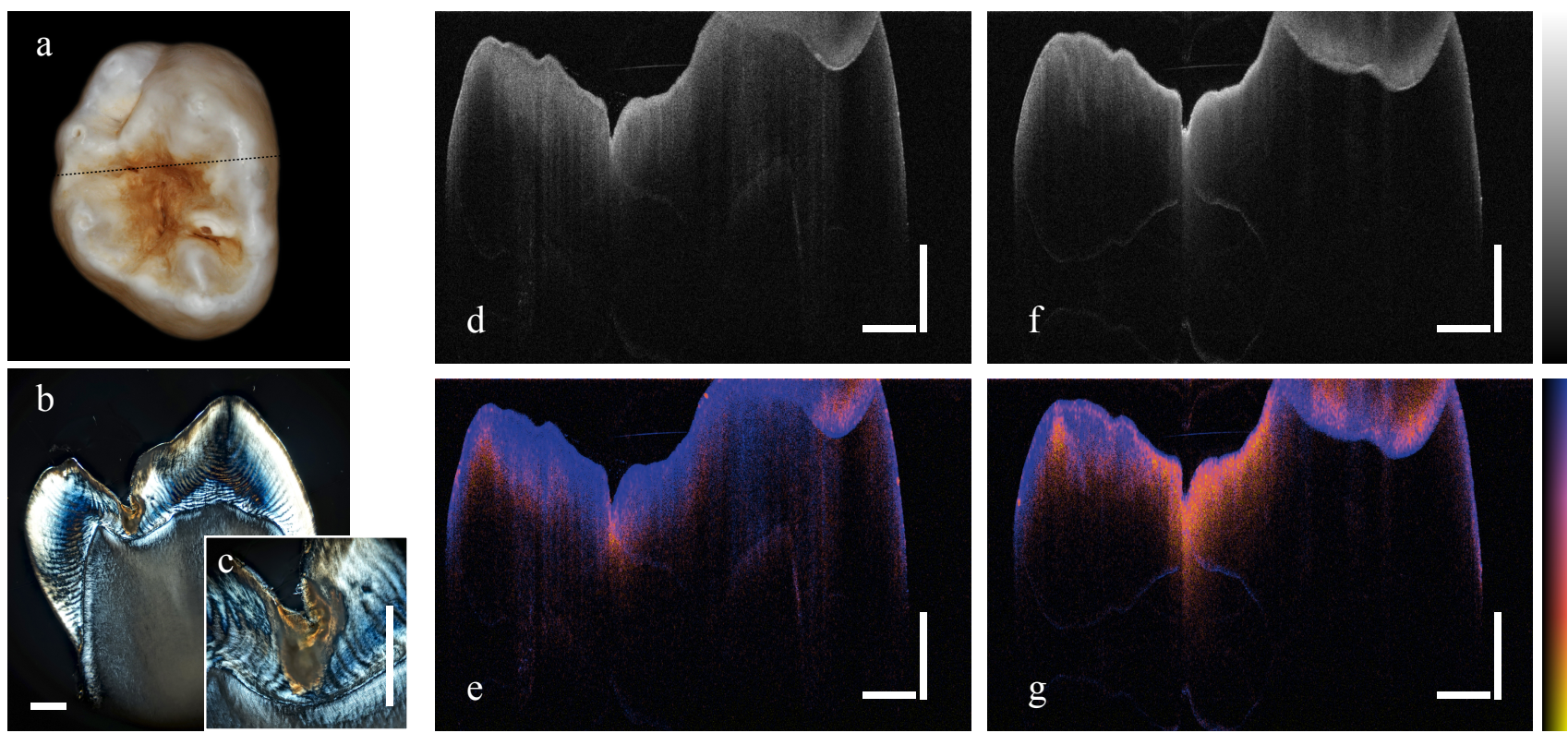

Figure 2. Visualizations of an occlusal carious lesion in the fissure: Photograph with marked slicing region (a), PLM images with 2.5x (b) and 10x (c) magnification, and PS-OCT images recorded under wet (d,e) and dry (f,g) conditions, displaying intensity $(\mathrm{d}, \mathrm{f})$ and depolarization $(\mathrm{e}, \mathrm{g})$. Color bars: intensity ranging from $3 \mathrm{~dB}$ (black) to $63 \mathrm{~dB}$ (white), depolarization from $\mathrm{DOP}=0$ (yellow) to $\mathrm{DOP}=1$ (blue). All scale bars: $1 \mathrm{~mm}$ in air.
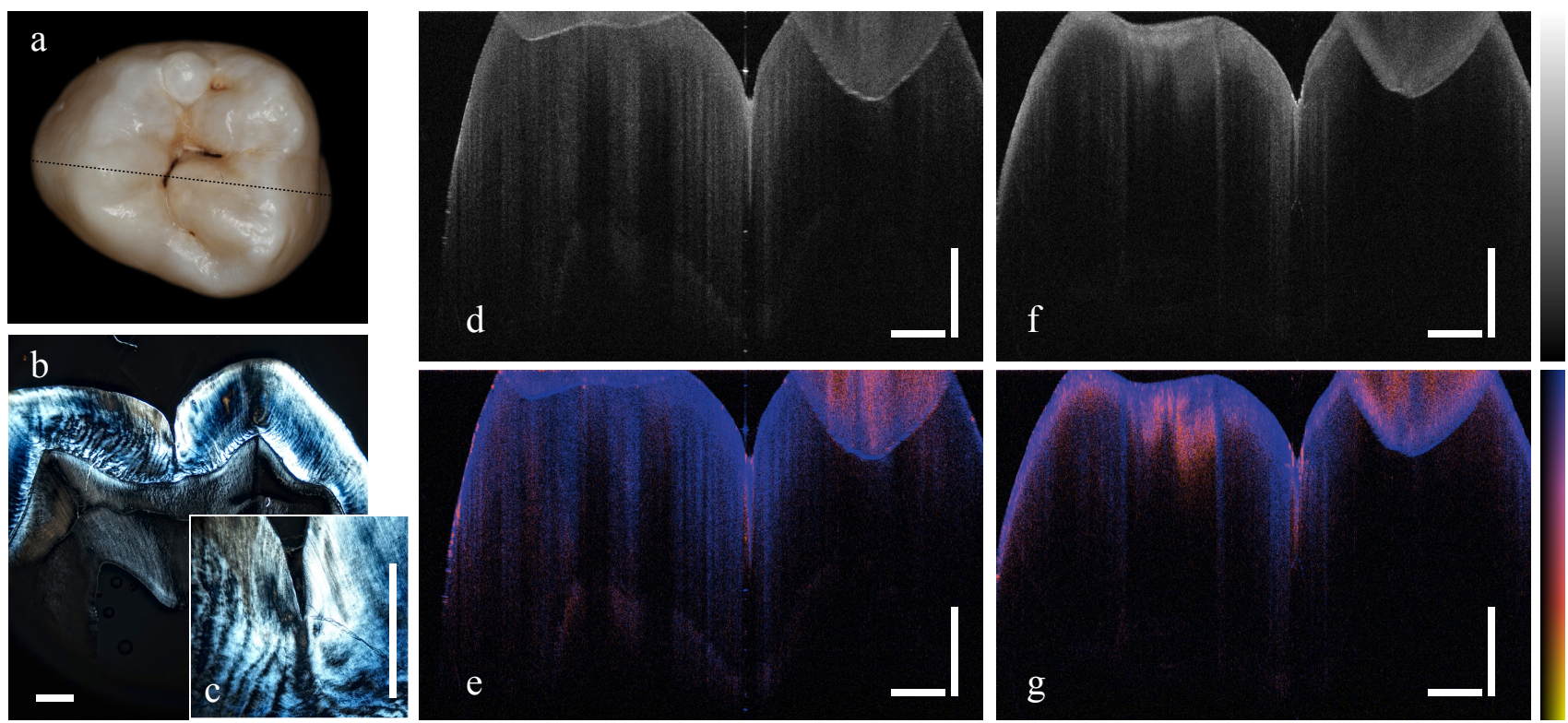

Figure 3. Visualizations of a molar tooth with stained fissures: Photograph with marked slicing region (a), PLM images with 2.5x (b) and 10x (c) magnification, and PS-OCT images recorded under wet (d,e) and dry (f,g) conditions, displaying intensity ( $\mathrm{d}, \mathrm{f})$ and depolarization $(\mathrm{e}, \mathrm{g})$. Color bars: intensity ranging from $3 \mathrm{~dB}$ (black) to $63 \mathrm{~dB}$ (white), depolarization from $\mathrm{DOP}=0$ (yellow) to $\mathrm{DOP}=1$ (blue). All scale bars: $1 \mathrm{~mm}$ in air. 
in comparison to sound enamel, whereas the depolarization indicates demineralization in the surrounding area of the fissure, presumably overestimating the existing lesion.

The second exemplary tooth in Fig. 3a shows clearly delimited dark discolorations in the fissures, which appear to be a superficial staining without impairment of the enamel in the region depicted by the PLM images $(\mathrm{b}, \mathrm{c})$. While both PS-OCT intensity images $(\mathrm{d}, \mathrm{f})$ for wet and dry recording indicate an altered scattering in the fissure, the depolarization representation corresponds to the PLM results without significant enamel alteration. Although thin sections of adjacent regions were not available, depolarization imaging indicated a demineralization in the unstained center of the fissure, as it is presented in Fig. 1 and was confirmed through the initial examination by a dentist.

In summary, depolarization imaging based on PS-OCT and with reduced noise dependency facilitates the differentiation of stained and demineralized enamel, and therefore, the assessment of occlusal alterations and lesions. Whereas this comparison of two samples implies a high dependency of the depolarization results on the hydration of the enamel with potential overestimation of the dry lesion, further investigation is necessary to evaluate the consequences for clinical translation of this technique.

\section{ACKNOWLEDGMENTS}

We want to thank Diana Jünger for preparing the thin sections. This project is supported by the European Union/European Social Fund (ESF) and the Free State of Saxony (project numbers 100270108 [FT, LK] and $100284305[\mathrm{JG}])$.

\section{REFERENCES}

[1] Schaefer, G., Pitchika, V., Litzenburger, F., Hickel, R., and Kühnisch, J., "Evaluation of occlusal caries detection and assessment by visual inspection, digital bitewing radiography and near-infrared light transillumination," Clinical oral investigations , 1-8 (2018).

[2] Ismail, A., "Visual and visuo-tactile detection of dental caries," Journal of dental research 83(1), 56-66 (2004).

[3] Almaz, E. C., Simon, J. C., Fried, D., and Darling, C. L., "Influence of stains on lesion contrast in the pits and fissures of tooth occlusal surfaces from 800-1600-nm," in [Lasers in Dentistry XXII], 9692, 96920X, International Society for Optics and Photonics (2016).

[4] Shimada, Y., Sadr, A., Burrow, M. F., Tagami, J., Ozawa, N., and Sumi, Y., "Validation of swept-source optical coherence tomography (ss-oct) for the diagnosis of occlusal caries," Journal of dentistry 38(8), $655-665$ (2010).

[5] Jones, R., Darling, C., Featherstone, J., and Fried, D., "Imaging artificial caries on the occlusal surfaces with polarization-sensitive optical coherence tomography," Caries research 40(2), 81-89 (2006).

[6] Golde, J., Tetschke, F., Walther, J., Rosenauer, T., Hempel, F., Hannig, C., Koch, E., and Kirsten, L., "Detection of carious lesions utilizing depolarization imaging by polarization sensitive optical coherence tomography," Journal of biomedical optics 23(7), 071203 (2018).

[7] Makita, S., Hong, Y.-J., Miura, M., and Yasuno, Y., "Degree of polarization uniformity with high noise immunity using polarization-sensitive optical coherence tomography," Optics letters 39(24), 6783-6786 (2014).

[8] Schmidt, W. J., Keil, A., et al., "Polarizing microscopy of dental tissues," (1971).

[9] Baumann, B., Augustin, M., Lichtenegger, A., Harper, D. J., Muck, M., Eugui, P., Wartak, A., Pircher, M., and Hitzenberger, C. K., "Polarization-sensitive optical coherence tomography imaging of the anterior mouse eye," Journal of biomedical optics 23(8), 086005 (2018).

[10] Golde, J., Tetschke, F., Walther, J., Rosenauer, T., Hempel, F., Hannig, C., Koch, E., and Kirsten, L., "Cross-sectional and en-face depolarization imaging for the assessment of dental lesions," Current Directions in Biomedical Engineering 4(1), 301-304 (2018). 